\title{
Research on Open Laboratory Construction Based on Internet of Things
}

\author{
Chunyu Mao, a ${ }^{1,}$ Mei Tian ${ }^{1, b}$ and Yanhong Sun ${ }^{1, c^{*}}$ \\ ${ }_{1}^{1}$ jilin Enjineerinfg Normal University, Changchun, China

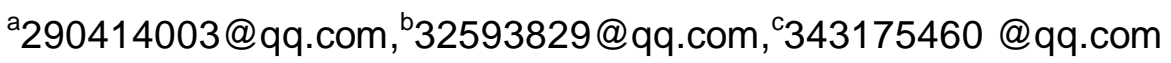 \\ * The corresponding author
}

Project source: "Exploration of Laboratory Construction of Mechanical and Electronic Engineering under the Background of Application-oriented Undergraduate Transition", Project of the Year of

"The 13th Five-year" Plan for Educational Science in Jilin Province, GH170630

\section{Keywords: Smart management; Internet of things; One card; Access control}

\begin{abstract}
In order to achieve open laboratory management, a laboratory intelligent management system design was proposed. The intelligent system adopts the Internet of Things technology as a whole and can carry out teaching and scientific research through the student and the teacher. The terminal can manage the lab comprehensively by authorizing a card. The intelligent system adopts the Internet of Things technology as a whole and can carry out teaching and scientific research through the student and the teacher. The terminal can manage the lab comprehensively by authorizing a card. The use of Internet of Things technology to achieve access control, attendance summary, appointment use, recording class process, results query and other functions. The open laboratory management based on the Internet of Things can improve the efficiency of laboratory use and achieve open laboratory management.
\end{abstract}

\section{Introduction}

In recent years, with the continuous deepening of higher education reform, especially under the promotion of "teaching evaluation" and "quality engineering", colleges and universities pay more attention to the cultivation of students' practical ability and innovation ability[1]. Open laboratories play an increasingly important role in cultivating innovative and applied talents. The laboratory is completely open, and it can integrate laboratory resources, increase the use of equipment and equipment, reduce the repeated acquisition rate of equipment and equipment, fully play the role of equipment efficiency, and increase investment efficiency[2]. In the past experimental teaching process, such a model of student participation in teacher research has been unconsciously opening up the laboratory[3]. "Opening up" is the subject of future laboratory work. Now we need to clarify our thinking, consciously implement regulations and regulations for the practice and exploration of open laboratory management, and provide a good teaching environment for experimental teaching. This is one of the basic conditions for running a higher education[4].

\section{Research Status}

In 2012, Wang Jiluo of Harbin Institute of Technology developed a smart home subordinate machine system based on embedded technology. The system can support multiple communication methods, connect to external networks through $\mathrm{Wi}-\mathrm{Fi}$, and have the ability to control multiple devices and turn things into things. Technology is applied to the development of the system[5]. In 2015, Zhang Minlei designed and implemented a laboratory management system based on the Internet of Things[6]. Through the network control equipment, the intelligent monitoring of the laboratory was realized. The system collects laboratory data through wireless nodes (ZigBee module, sensor module, and alarm module), monitors temperature, humidity and other information in the laboratory, adopts automatic alarm mechanism, NFC card reader authentication, mobile phone App control indoor lighting, etc. Ways to achieve automated laboratory management and intelligent monitoring. Applying technologies such as cloud computing, virtualization, and the Internet of Things in laboratory management is a new 
trend in laboratory construction[7]. Build a cloud computing platform to concentrate business performance computing resources on the server side for unified management, which is conducive to the rational allocation of computing resources and the improvement of computing service quality. The use of wireless nodes to monitor all data in the laboratory in real time reduces the burden on managers[8].

\section{Research on the Construction of Open Laboratory Based on Internet of Things}

The construction of open laboratories needs to be continuously summed up in practice, and its ultimate realization cannot be separated from technical support. Today, IOT technology is increasingly permeating every corner of life. Not only open laboratories, but also smart campuses (intelligent collection, transportation, management, control, display, push, etc. of campus information, and eventually the entire school's departments, subsystems information integration, interconnection and interoperability) are based on the Internet of Things cloud Calculation is the core. The open laboratory technology based on the Internet of Things can effectively solve the problems of lagging information renewal in laboratories, shortage or waste of human resources, equipment redundancy, repeated input, etc., and can also effectively achieve the scientific decision-making that open laboratories need. Fully open, timely control, convenient and quick requirements[9].

Hardware Structure Design of Open Experiment Management System. The open laboratory hierarchy architecture model uses a three-tier structure similar to that of the Internet of Things. From top to bottom, the sensor layer, network layer, and integrated application layer are shown in Figure 1.

Network Layer Construction. As the middle layer of the Internet of Things, the Internet layer, with the help of the Internet, wireless broadband, and telecom backbone networks, carries important tasks such as access, transmission, and operation of sensing data. The Internet layer of the Internet of Things may be built after "multi-network convergence." Above the backbone network, it may also be various types of private networks. The Internet of Things technologies involved in the network layer include IOT nodes and gateway technologies, IOT communication and frequency management technologies, and IOT access and networking technologies. Existing campus networks, WLANs, and mobile 2G/3G networks in the laboratory and wireless sensor networks built for the laboratory will become the main body of the network layer. The network entities work together and demonstrate their capabilities[10].

Perception Layer Construction. The sensory layer relies on a large number of sensing devices distributed in the laboratory to collect and transmit various types of data information to the network layer. These devices mainly include RFID devices, sensor devices, and smart M2M terminals. As the core technology in the concept of the Internet of Things, RFID has a very wide range of applications, and it can fully apply its successful experience in the field of logistics to the construction of open laboratories. The device that integrates the RFID read-write chip can serve as the main body of the sensing layer and cooperate with a variety of sensor devices to form a sensory network covering the entire laboratory. Among the existing sensing devices, RFID devices have obvious advantages and play a greater role. Among them, low-frequency RFID devices can be used for applications such as credit card attendance and identification, and high-frequency RFID devices can be used in the supervision of laboratory equipment through laboratory-wide multi-tag identification technology. RFID devices should be designed to be wired and wirelessly connected to the laboratory network and upload the collected basic data to the server.

Application Layer Construction. The perception layer and network layer ultimately serve the application layer. It is the application of a large number of reliable, fast, and intelligent service applications that demonstrate the wisdom of the laboratory. The application layer aims to build a multi-service integrated platform and realizes the integration of wisdom in many applications such as teaching management, resource management, and environmental monitoring. They will no longer be independent and separate structures, but integrated on a unified platform. Form an open laboratory system.

Software Design of Open Experiment Management System. Design of experiment management subsystem for IOT course. For the open lab, the course experiment management is the experimental content required by the traditional syllabus. After connecting it to the Internet of Things, the teacher of 
the experiment class can make an appointment from the teacher's site and time for the experimental course of the lesson and upload the content and steps of the experiment. Students can preview the content uploaded by the teacher from the student side. The specific system design is shown in Figure 2 - before the experiment. The design function: access control management can authorize students to open the card access control; attendance summary can automatically generate a time and attendance table for attendance staff statistics (instead of students reported to attend classes); video management can be real-time video recording preparation class process, see Figure 2 - experiment[11].

The Internet of Things technology is used in the course experiment management to facilitate the improvement of management efficiency and the intelligent collection of information. Pre-classroom preparation, practice in class, and after-class report submission are all documented to improve learning efficiency. Instruments and equipment are authorized by the supervision and use of the teacher, and the equipment usage records are automatically established to facilitate data statistics. Responsibility can be fulfilled and the workload of the experimenter can also be reduced. The design function: to achieve the appointment of the open laboratory use of the right (reservation time, experiment content and location); authorized student card, students can enjoy the right to open the laboratory during the appointment time period to complete the independent experiments. The design advantages: Design can reduce the burden on teachers, improve student hands-on capabilities; Automatic establishment of equipment usage and device consumption records, to facilitate data statistics and analysis; Improve the utilization rate of open laboratories, improve the equipment usage rate, equipment account management (Maintenance, Maintenance, Retirement) Provides the basis; Video Surveillance Provides Security for Open Labs.

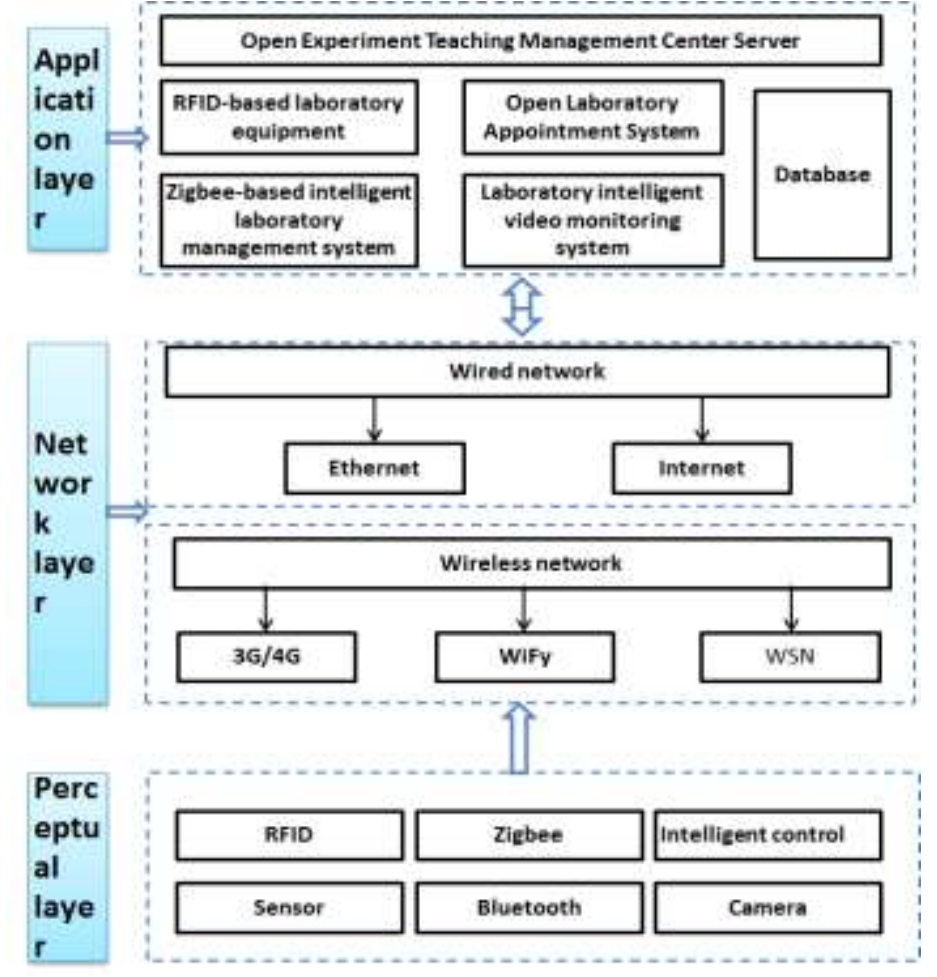

Figure 1. Open Lab Hardware System Block Diagram

The Design of Open Laboratory Equipment based on the Internet of Things. The Internet of Things technology is used for the management of laboratory instruments and equipment. Data is collected and recorded in real-time on the use of instruments and equipment. Eventually, real-time management of equipment and instruments can be realized throughout the life cycle of purchase, use, maintenance, maintenance, and retirement.

The equipment management system based on the Internet of Things mainly includes: user management, procurement process management, acceptance flow management, use process management, valuable equipment and instrument management, maintenance management, end-of-life management, and supplier management. 
IOT-based Instrument and Equipment User Management. The school's laboratory management base (including the director, laboratory director, and laboratory technician) can enter the system to inquire, manage, and track the equipment.

Based on the Internet of Things Equipment Procurement Management. Relevant personnel responsible for procurement of equipment and equipment register procurement items and bidding information on the Internet of Things to make the procurement process transparent and open.

IOT-based Equipment and Equipment Acceptance Process Management. After the acceptance of the organization, the equipment is registered on the Internet of Things and an RFID tag is affixed to automatically form a report (including the name, model, quantity, picture, supplier information, etc. of the equipment).

IOT-based Instrument and Equipment Use Process Management. Through remote online collection of equipment and equipment use time, frequency, personnel and other information, record equipment and equipment usage, whether damaged, illegal operation, whether there is maintenance records.

Based on the Internet of Things, Valuable Equipment and Equipment Management. Mainly for large-scale precious equipment and equipment for the external use rate, borrow records, authorized use, care management.

IOT-based Instrument and Equipment Maintenance Management. If the equipment needs maintenance, the person in charge of use at the time is registered in the record of the Internet of Things from the application maintenance, maintenance registration, and maintenance end.

IOT-based Instrument and Equipment Retirement Management. If the equipment needs to be scrapped, the management personnel can record the information such as scrapping application, scrap evaluation, and scrap cancellation.

IOT-based Instrument Equipment Supplier Management. Record suppliers' contract information, equipment's advantages and disadvantages, and after-sales service information to provide reference for the school's next purchase of equipment.

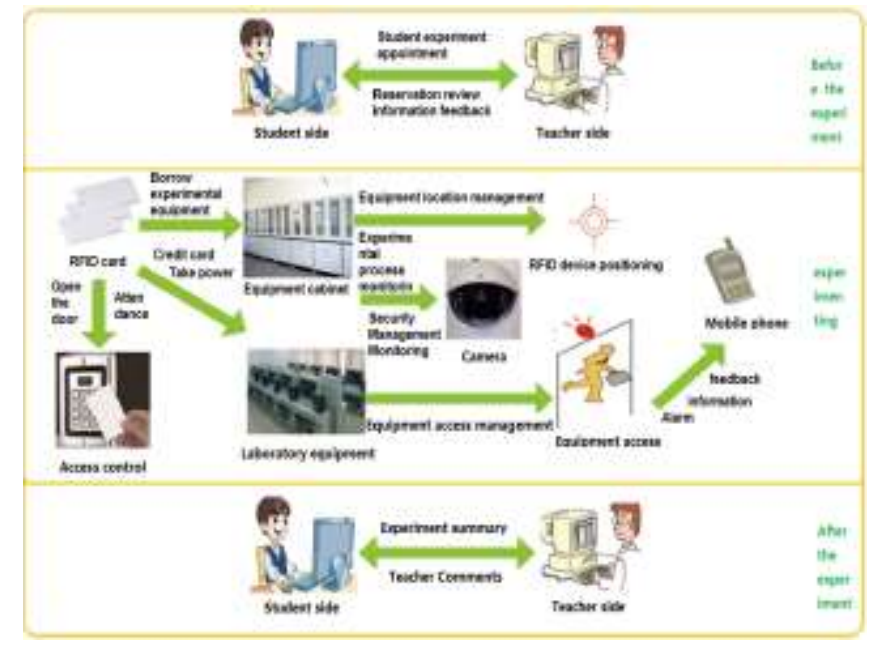

Figure 2. Open Lab Management System Block Diagram

\section{Summary}

With the deepening of teaching reform in colleges and universities, the open experimental teaching mode will become the mainstream of experimental teaching. Practice has proved that the full opening of the electronic laboratory for mechanical and electronic engineering is conducive to cultivating students' innovative ability and improving the overall quality of students. However, the reform of open experimental teaching is a long-term and arduous systematic project. It is necessary to constantly explore experimental projects, teaching methods, laboratory management, and faculty, in order to form a scientific, rational, and effective opening. The new experimental teaching mode will promote the 
improvement of the experimental teaching level in our country's colleges and universities, and explore a practical experimental teaching path that has the characteristics of the school and meets the requirements of mechanical and electronic engineering students.

\section{References}

[1] HUANG Zheng,GU Peng.Research and Discussion on the Construction of Internet of Things Laboratory[J]. Experimental Technology and Management. 2012(02).

[2] Bai Yuhong. A Comparative Study of the Dual-Teacher Construction of the "Combination of Work and Study" Model in Higher Vocational Education [J]. Higher Education Exploration, 2008, (4) 57-61.

[3] Hong Bin, Chen Xiaofei. Discussion on the Construction Mode of University Laboratories under New Form[J]. Research on Laboratory Work in Colleges and Universities, 2008, 3:101-104.

[4] Chai Lihong. Preliminary Study on the Construction of Internet of Things Specialty Laboratory in Higher Vocational Colleges[J]. China Science and Technology Expo, 2012(2):94-94.

[5] Wang Haichun, Yu Long, Li Jun. Exploration of New Type Internet of Things Laboratory Construction Project in Colleges and Universities[J]. Education Education Forum, 2015(52): 240-241.

[6] K. B. Hendricks, V. R. Singhal, J. K. Stratman. The impact of enterprise Systems on corporate performance: a study of open aboratory management system Implementations[J]. Journal of operations management, 2011, 25(2):65-66.

[7] Sookhak M, Gani A, Talebian H, etet al. Remote data auditing Survey, taxonomy, and open issues[J]. ACM Computing Surveys (CSUR), 2015, 47 (4): 65.

[8] D.W.Vanvuren. An empirical investigation of the effectiveness of Enterprise resource planning systems, as assessed by management accountants[M]. America: the university of miss apply, 2010: 76-77.

[9] Helen Staer, Denis goodrum and mark hackling. High school laboratory work In western australia: openness to inquiry[J]. Research in science education, 2011, 28(2): 237-240.

[10] Timmothy Posey, Wyatt Barnett, Cristian Darie.Build Your Own ASP.NET 4 Web Site Using C\# and VB[M].Sitepoint Pty Ltd,2011,198-199.

[11] Kalen Delaney,P. Randal, Kimberly L. Tripp.Microsoft SQL Server 2008 Internals[M]. Microsoft Press,U.S,2010,689-692. 